\title{
UNA APROXIMACIÓN \\ A LAS INTERVENCIONES \\ DISCRECIONALES DEL ESTADO \\ COMO FUENTE DE INESTABILIDAD \\ FINANCIERA
}

ANTONIO PANCORBO DE RATO*

Resumen: Son muchas las voces que reclaman una revisión del diseño del sistema financiero actual, capaz de provocar una crisis mundial como la que ahora vivimos. Sin embargo, pocas de estas voces ven necesario que se revise el papel activo y discrecional del Estado para fomentar, salvaguardar y recobrar la estabilidad financiera. Este artículo pretende ser un contrapunto en este debate. Desde la hipótesis de que las actuaciones discrecionales del Estado son una causa endógena con capacidad para desorientar al sistema en su conjunto y provocar crisis sistémicas, el artículo apunta de forma introductoria el rediseño necesario de las principales instituciones monetarias y financieras (dinero, autoridades monetarias, modelo de negocio bancario, entre otras) que elimine dicha intervención discrecional. La actuación del Estado quedaría limitada a asegurar que las fuerzas dispersas del mercado actúen libre y responsablemente respetando la ley, la propiedad y los contratos. El artículo concluye con los posibles efectos que tendría dicho rediseño institucional para la estabilidad financiera. Desde esta primera aproximación, estos efectos serían beneficiosos lo que animan a continuar esta línea de investigación.

Palabras clave: Estabilidad Financiera, Intervención del Estado, Banca, Dinero.

Clasificación JEL: E42, E52, E58, G21.

* Doctor en Economía, actualmente desempeña sus funciones en el Fondo Monetario Internacional (www.imf.org), procedente del Banco de España (www.bde.es). Anteriormente fue miembro de la Secretaría del Comité de Supervisores Bancarios de Basilea (Banco de Pagos Internacionales, www.bis.org). Las opiniones que se expresan en este artículo son exclusivamente las del autor (apancorbo@imf.org) 
Abstract: Many have called for the design of the present financial system -one capable of causing the global crisis currently assailing us - to be overhauled. Yet few feel that the State's active and discretionary role needs to be changed so as to promote, safeguard and shore up financial stability. This article aims to act as a catalyst in this debate. On the assumption that State discretionary actions are an endogenous factor capable of upsetting the system as a whole and prompting systemic crises, the article makes a case for re-designing the main monetary and financial institutions (money, monetary authorities, banking business model, inter alia) to eliminate such discretionary intervention. State conduct would be confined to ensuring that diffuse market forces act freely and responsibly, observing the law, property rights and contracts. The article concludes with the potential effects this institutional redesign would have on financial stability. Tentatively, these effects would be beneficial, suggesting this avenue of research should be pursued.

Key words: Financial Stability, State Intervention, Banking, Money.

JEL Classification: E42, E52, E58, G21.

INTRODUCCIÓN: EL INTERÉS CRECIENTE DEL SECTOR PÚBLICO POR LA ESTABILIDAD FINANCIERA

La estabilidad financiera es un prerrequisito para el crecimiento económico, por tanto, debería ser un objetivo para cualquier sociedad que quiera estar positivamente al servicio del ser humano. En efecto, la contribución crítica del sistema financiero a la promoción del «bien común», 1 por sí, es un argumento suficiente para justificar el interés creciente del sector público por la estabilidad financiera. Sin embargo, fue la sucesión de crisis bancarias nacionales durante el tercer cuarto del siglo $X X, y$ con impacto creciente en términos de coste fiscal, lo que despertó el interés de las autoridades nacionales y la comunidad financiera internacional por la estabilidad financiera. Este interés se vio reforzado

1 Se entiende por «bien común» el conjunto de condiciones de la vida social y económica que hacen posible a las asociaciones y a cada uno de sus miembros el logro más pleno y más fácil de su propio bien individual. 
con la ocurrencia de crisis bancarias en países emergentes, pero con efectos sobre el sistema financiero internacional, como las crisis de Latinoamérica de mediados de los $90 \mathrm{y}$, sobre todo, las crisis del sudeste asiático de finales de los 90. La actual crisis económica mundial, que se iniciase en el verano de 2007, ha sido sin duda el revulsivo determinante. La «arquitectura» del sistema financiero que surgió tras las crisis bancarias de los años 80 y 90, y que se concibió, precisamente, para evitar y contener futuras crisis de naturaleza sistémica, ha permitido, sin embargo, que tenga lugar con rapidez y virulencia el mayor colapso del sistema financiero internacional que jamás se haya visto, poniendo en serio peligro las posibilidades de crecimiento económico. A estos efectos sobre la economía, habría que añadir los altos costes políticos que suelen venir asociados a toda crisis financiera para entender por qué hoy los temas de estabilidad financiera son una prioridad en la agenda política internacional.

Frente al interés creciente por la estabilidad financiera y amplia dedicación de recursos por parte del sector público, llama la atención el escaso interés por parte del sector privado. Más aún teniendo en cuenta que es precisamente el sector privado quien más sufre las consecuencias de las situaciones de crisis financieras. Una posible explicación de este menor interés puede ser que la actuación del sector público para salvaguardar la estabilidad financiera se considera suficiente. Otra posible explicación, no excluyente de la anterior aunque más perniciosa, sería que el excesivo énfasis puesto por el sector público en salvaguardar la estabilidad financiera es aprovechado por el sector privado para tomar posiciones más arriesgadas y confiar en el rescate público (gambling for the rescue). Esto introduciría el debate sobre el riesgo moral (moral hazard) que ha sido frecuentemente mencionado en la actual crisis económica. Una última explicación, tampoco excluyente con las anteriores, sería que el sector privado ve los temas y las necesidades relacionadas con la estabilidad financiera de forma diferente atendiendo más a sus necesidades de mercado. En este sentido, no se puede decir que no haya interés por parte del sector privado, sino que el método de trabajo y enfoque de estudio es distinto al que sigue el sector público centrado en las funciones de las distintas instituciones públicas implicadas 
en la salvaguarda de la estabilidad financiera (normalmente, el banco central, los supervisores prudenciales, los esquemas de garantías de depósitos y los ministerios de finanzas).

Por último, un argumento frecuente para justificar el interés creciente del sector público por la estabilidad financiera es considerarla un «bien público». ${ }^{2}$ La idea de estabilidad financiera como bien público se refuerza aún más cuando se contemplan las «externalidades negativas» que se producen en situaciones de inestabilidad financiera, ya sea en los mercados o en las instituciones financieras individuales. También, la aparente falta de interés del sector privado por la estabilidad financiera vendría a reforzar su consideración como un bien público (Schinasi, 2006). Sin embargo, no debería ser doctrina pacífica concluir que, ya que la estabilidad financiera es un posible «bien público», esto justifique la intervención del Estado. Habría que responder primero a qué tipo de estabilidad financiera se pretende conseguir y cómo la puede conseguir el Estado. Como veremos a continuación, uno de los principales problemas es la imposibilidad de encontrar una definición consensuada de estabilidad financiera, por lo que se pretendería una intervención del Estado para producir un supuesto «bien público» que no se es capaz de precisar. La falta de una definición consensuada y operativa de estabilidad financiera también dificulta la labor de identificar qué tipo de intervención se espera del Estado. Prácticamente cabe todo tipo de intervención pública bajo un concepto, hoy ubicuo, de estabilidad financiera.

2 Schinasi (2006) expone una buena argumentación desde el punto de vista de escuelas de pensamiento keynesiano, de por qué el dinero, las finanzas y la estabilidad financiera son bienes públicos. La teoría económica dominante entiende que un bien económico será un bien público (v.gr.: la defensa nacional), en contraposición con los bienes privados (v.gr.: los alimentos), si no hay rivalidad en su consumo, es decir, que el consumo por parte de un individuo no reduce la cantidad disponible para otros, y si es imposible evitar, por medios físicos o legales, que un tercero que no haya contribuido en sus costes de producción se pueda beneficiar de dicho bien. 


\section{II \\ LA ESTABILIDAD FINANCIERA COMO OBJETIVO DE POLÍTICA PÚBLICA}

\section{1. ¿Qué se puede entender por «estabilidad financiera»?}

La estabilidad financiera es el tema central de infinidad de artículos, seminarios, conferencias y trabajos, $\mathrm{y}$, sin embargo, es un término que no cuenta con límites precisos. Tanto las autoridades públicas como el mundo académico han ofrecido una gran variedad de definiciones que van desde una definición estrecha de estabilidad financiera entendida como la ausencia de crisis, a una definición amplia que la consideraría como la capacidad de un sistema financiero para promover el desarrollo económico. Al hablar de estabilidad financiera siempre nos moveremos dentro de un concepto amplio y difuso, pero, por otra parte, esencial para el bien común de cualquier sociedad.

Intuitivamente, la estabilidad financiera es una situación en la cual las instituciones, mercados e infraestructuras del sistema financiero funcionan como es debido. Profundizando un poco más, el hecho diferenciador de una situación de estabilidad financiera frente a una de inestabilidad es que los agentes económicos tienen confianza en las instituciones, mercados e infraestructuras del sistema financiero. Esta idea de estabilidad financiera como situación de confianza, sin ser muy precisa, permite al menos orientar el debate e identificar ciertos prerrequisitos que sienten las bases para que esta confianza de los agentes en el sistema financiero se vea fortalecida:

- Un marco legal que dé seguridad jurídica a los contratos y los derechos de propiedad.

- Un marco institucional sólido y eficiente, tanto por parte de las instituciones públicas como privadas.

- Unas políticas económicas sanas, dirigidas a alcanzar estabilidad monetaria y disciplina fiscal.

- Un comportamiento de los agentes enraizado en sólidos fundamentos éticos. 
- Unas autoridades financieras que gocen de reconocido prestigio y competencia profesional.

De hecho, periodos de relativa bonanza económica y financiera no garantizan que se esté en una situación de estabilidad financiera si llegados periodos de turbulencia y crisis financiera estos prerrequisitos se perdieran. Evaluar una situación de estabilidad financiera solo por síntomas externos, como indicadores de mercado, nos puede llevar a la «paradoja de la estabilidad financiera», según la cual un sistema financiero se puede mostrar estable, cuando es más vulnerable. La estabilidad financiera se debe determinar por la solidez de las bases sobre las que el sistema financiero está construido. De hecho, episodios de crisis en entidades individuales o altas volatilidades no son menoscabo para la estabilidad financiera, siempre y cuando las instituciones, mercados e infraestructuras del sistema financiero continúen funcionando como es debido, permitiendo una adecuada asignación y movilización de los recursos financieros.

\section{La necesidad de una «definición operativa»}

La ausencia de una definición precisa de estabilidad financiera, en principio, no sería un problema en sí. No todos los conceptos cuentan con una definición precisa con consecuencias prácticas. El amor, la libertad o la paz, entre otros muchos, cuentan con un grado de interpretación subjetiva que no les resta por ello ningún valor en el desarrollo del pensamiento. El problema de la indefinición del concepto de «estabilidad financiera» se deriva de constituir una finalidad de la intervención del Estado en la economía. Justificar la intervención del Estado sobre conceptos ambiguos puede ser causa de ineficiencia en su actuación o de una intromisión más allá de aquello para lo que el Estado está legitimado.

¿Es posible una definición operativa de estabilidad financiera sobre la que sea posible articular un objetivo de política pública? Si bien las definiciones que ofrecen las distintas autoridades públicas permiten delimitar una serie de expectativas sobre lo que se espera de un sistema financiero estable (que tenga capacidad 
para asignar recursos, prestar servicios financieros de forma eficiente y ordenada, generar confianza, o absorber posibles acontecimientos adversos), estas definiciones no nos permiten justificar un alcance de la intervención del Estado en el sistema financiero que vaya más allá de asegurar la ley y el orden. Sin embargo, cualquier intervención que el Estado desease realizar en el sistema financiero está justificada en tanto no se cuente con una definición no operativa, con el riesgo que esto supone de una intervención demasiado fuerte que amenace la libertad, la iniciativa personal y la competencia.

Queda pendiente, por tanto, la tarea de elaborar una definición operativa de estabilidad financiera que pueda dar respuesta, entre otras, a las siguientes preguntas. ¿Cuál es el objetivo público de estabilidad financiera? ¿Es suficiente para alcanzar este objetivo la solvencia de las entidades individuales y el buen funcionamiento de las estructuras financieras? ¿Se puede alcanzar en todo o en parte este objetivo mediante incentivos de mercado y las actuaciones de las asociaciones profesionales? Aún así, ¿es conveniente la intervención del Estado? ¿Qué medidas de intervención pública serían las apropiadas?

III

\section{LAS ACTUACIONES DEL ESTADO PARA LA SALVAGUARDIA DE LA ESTABILIDAD FINANCIERA}

Los efectos crecientes de las crisis financieras sobre el conjunto de la economía la mayor recurrencia con que suceden y los costes políticos que llevan asociados, han motivado que los poderes públicos, de forma cada vez más intensa e intrusiva, busquen diseñar medidas públicas para mitigar o eliminar los efectos de las crisis financieras y reducir su ocurrencia. Estas actuaciones del Estado, lo que se ha venido en llamar las «redes de seguridad», incluyen, en primer lugar, la regulación y supervisión prudencial, como actuaciones preventivas encaminadas a establecer un marco de solidez en el sistema financiero y a la identificación temprana de aquellos riesgos que puedan ser causa de su mal funcionamiento. 
Junto con estas actuaciones, la actuación del banco central como prestamista de última instancia y los fondos de garantía de depósitos buscan reforzar la confianza de los agentes económicos en el sistema financiero. Por último se encuentran los mecanismos de resolución de crisis bancarias para aquellas entidades que no son capaces de cumplir con las medidas prudenciales impuestas por los organismos públicos de control. Así, el sistema financiero, y en especial el sector bancario, son actividades económicas fuertemente intervenidas por el Estado. Entender lo contrario sería un error serio en la percepción de la realidad.

\section{La regulación y supervisión prudencial}

La teoría económica dominante justifica la supervisión y regulación prudencial fundamentalmente por el coste social de las externalidades que genera una crisis bancaria. La regulación financiera busca la identificación de «fallos de mercado» que dicha regulación vendrá a corregir. ${ }^{3}$ Con este fin, los poderes públicos ponen especial énfasis en diseñar regulaciones estrictas que aseguren la calidad de los activos, la situación de liquidez, las prácticas de gestión, la rentabilidad y la capitalización de las instituciones financieras. La supervisión prudencial busca hacer cumplir esta regulación con el fin de mitigar el riesgo sistémico y de proteger a los consumidores (depositantes).

La regulación y supervisión prudencial no se plantean, en principio, como garantías contra las crisis financieras. Si bien se entiende que una regulación y supervisión efectivas son factores

3 La teoría económica dominante entiende por «fallo de mercado» la situación que se produce cuando el suministro que hace el mercado de un bien o servicio no es eficiente, o éste produce externalidades negativas. La existencia de «fallos de mercado» justificaría la intervención del Estado para corregirlos, ya que se entiende que su actuación proactiva y coercitiva producirá resultados más eficientes que los que resultasen de los intercambios voluntarios en un régimen de libre mercado. Posiciones críticas con esta doctrina, por el contrario, entienden que es el propio mercado el que se perfecciona al detectar los fallos, identificar oportunidades de beneficio $y$, de este modo, encontrar en un proceso de prueba y error soluciones eficientes (ver, por ejemplo, Guillory, 2005). 
importantes que ayudan a reducir la ocurrencia y los efectos de las mismas. El supervisor tratará de adelantarse e identificar aquellas practicas incorrectas y situaciones insostenibles que dejadas al juego del libre mercado supondrían la quiebra de esas entidades, pero que, dada la naturaleza social e imprescindible del sistema financiero, tales quiebras no serían socialmente admisibles. Tanto es esto así que, en la práctica, se puede llegar a entender que la regulación y supervisión financiera han suplantado en buena parte a la disciplina de mercado. El mercado baja la guardia ante la presencia del supervisor. Pero también esta suplantación de las leyes del mercado ha aumentado la responsabilidad implícita del supervisor cuando fracasa a la hora de identificar y resolver situaciones de crisis, lo que ocasiona que una crisis financiera sea también una crisis para el propio supervisor.

\section{El banco central como prestamista de última instancia}

La doctrina tradicional asocia la función de prestamista de última instancia del banco central con la prevención y la mitigación de las crisis financieras. Se justifica la existencia del prestamista de última instancia por la necesidad de cubrir un «fallo de mercado» que hace que una entidad con un problema de liquidez, que no de solvencia, no pueda obtener en los mercados interbancarios la liquidez necesaria para atender sus obligaciones. El prestamista de última instancia permite a los bancos financiar a corto plazo, incluso con depósitos a la vista, inversiones a plazos más dilatados, y más rentables. La doctrina dominante entiende que esto debe ser así dada la «función social» de los bancos de intermediación en los plazos. De este modo, la operativa de los bancos goza del privilegio de acceso a la liquidez ilimitada del banco central como modo de proteger sus estructuras financieras inestables contra los pánicos bancarios.

En sus actuaciones como prestamistas de última instancia, es común que los bancos centrales se adecuen a la llamada «doctrina Bagehot» (Bagehot, 1873), según la cual el banco central en situaciones de falta de liquidez, y con el fin de no incurrir en coste fiscal recapitalizando entidades, solo debe actuar como prestamista 
de forma ilimitada con entidades solventes, aplicando un tipo de interés penalizador y contra la aportación de garantías suficientes. Los problemas prácticos, sin embargo, surgen en el momento de definir qué es un banco solvente y qué son garantías suficientes. La actual crisis financiera mundial ha hecho necesario rebajar temporalmente los requisitos de la doctrina tradicional.

\section{Los sistemas de garantía de depósitos}

La mayor parte de los sistemas bancarios modernos cuentan con un sistema de garantía de depósitos. Los fondos de garantía de depósito (las instituciones públicas y en ocasiones privadas o de gestión mixta en que se materializan) se constituyen con el fin de garantizar una fracción o la totalidad de los depósitos en caso de la suspensión de pagos del banco. El objetivo último de los sistemas de garantía de depósitos es doble, por un lado, aseguran la confianza de los depositantes en el sistema bancario que eviten pánicos por anuncios o rumores sobre problemas de solvencia o liquidez en uno o más bancos. Por otro, rebajan sustancialmente las necesidades de evaluación de los bancos por parte de los depositantes garantizados, a quienes la doctrina dominante atribuye una capacidad muy escasa para determinar la confianza que les merecen las distintas instituciones bancarias. ${ }^{4}$

Los sistemas de garantía de depósitos, a diferencia de las funciones de prestamista de última instancia, aseguran contra la insolvencia de los bancos, no contra su iliquidez. Es precisamente la presencia de los problemas de riesgo moral lo que hace tan importante el diseño adecuado de los sistemas de garantía depósitos. La experiencia internacional demuestra que si bien un seguro de depósitos puede contribuir a la estabilidad financiera, un diseño deficiente en el que se materialicen los problemas de riesgo moral y selección adversa puede llevar a crisis sistémicas. También

\footnotetext{
${ }^{4}$ Los fondos de garantía de depósitos también pueden tener como función y objetivo ser parte de la estructura institucional destinada a la reestructuración o el cierre de un banco insolvente de forma ordenada, estableciendo procedimientos explícitos para acceder a los recursos del fondo.
} 
demuestra que los sistemas de garantía de depósitos cumplen sus funciones de estabilidad de forma eficiente en periodos de relativa normalidad del sistema bancario. En periodos de crisis sistémicas su capacidad se ve considerablemente mermada por los propios problemas de financiación de los fondos. Los gobiernos generalmente se ven obligados a cubrir las garantías y expandirla a la totalidad de los depósitos para evitar un pánico generalizado, lo que implica una nueva partida de coste fiscal para la salida de la crisis. Una vez que se está ante el riesgo de colapso generalizado del sistema bancario, la cuestión se convierte en un problema fiscal. Un fondo de garantía de depósitos difícilmente podrá cubrir los compromisos asumidos en un sistema bancario nacional. Su función es más bien tratar de desincentivar los pánicos bancarios antes que éstos sucedan.

\section{Los procesos de intervención y resolución de bancos}

Cuando un banco no es capaz de cumplir con los requerimientos de la regulación prudencial, las autoridades financieras toman las medidas necesarias para que la resolución de esta situación se efectúe de manera ordenada, procurando preservar el valor de los activos, la estabilidad de los depósitos y del sistema de pagos. Para estos casos, las autoridades financieras desarrollan unos mecanismos especiales de resolución de crisis bancarias que suelen incluir facultades para tomar el control y la administración del banco en crisis, antes incluso de que se produzca la insolvencia técnica, y poner en marcha un plan de reestructuración bancaria. Puede suceder que dada una situación de insolvencia bancaria, se decida liquidar el banco en lugar de rescatarlo. Sin embargo, según el estudio de Goodhart y Shoenmaker (1995), en la mayoría de los casos estudiados las autoridades financieras optaron por el rescate de los bancos. En estos casos, además del coste fiscal asociado con la medida, hay que tener en cuenta el riesgo moral que se introduce; así, se vuelve a reforzar la idea que comúnmente se atribuye a Walter Bagehot: «una ayuda hoy a un banco malo es la mejor manera de evitar el establecimiento de un banco bueno en el futuro». 
El tratamiento de las resoluciones de crisis bancarias cuenta con una complejidad adicional para aquellos bancos que se entienda que tienen una importancia sistémica (que se consideren «too-big-to-fail» en la terminología anglosajona donde se acuñó este término). Estas entidades gozarían de una garantía adicional implícita por parte de los poderes públicos, garantía que también en cierto modo está descontada por los mercados financieros, por las agencias de calificación e incluso por los propios altos cargos de las entidades.

IV

\section{NECESIDAD DE REVISAR EL PARADIGMA DOMINANTE}

A pesar de todos los esfuerzos de los poderes públicos para salvaguardar la estabilidad financiera, el sistema financiero es cada vez más inestable. Nunca hasta ahora los gobiernos en economías de mercado habían tenido que tomar las medidas sin precedentes de apoyos de liquidez y estímulos fiscales que se han venido tomando desde el cuarto trimestre de 2008,5 pese a las situaciones verdaderamente dramáticas que se han vivido en el siglo $X X$ en tiempos de paz. Voces destacadas piden la revisión del diseño actual del sistema financiero. Sin embargo, prácticamente todas estas voces no cuestionan las intervenciones del Estado para la salvaguarda de la estabilidad financiera que acabamos de ver, o incluso piden que se refuercen en el mismo sentido.

Las crisis financieras, como la actual crisis mundial, son, ante todo, un producto de la mala gestión de las entidades. Sin embargo, sorprende que prácticamente todas las entidades de crédito hayan cometido errores en la misma dirección y al mismo tiempo.

5 Sin ánimo de ser exhaustivo, estas medidas extraordinarias y sin precedentes se pueden resumir en el aumento de las garantías a los depósitos del público en las entidades bancarias; la concesión de avales o garantías públicas a las emisiones de deuda realizadas por los bancos; la capitalización de los bancos con fondos públicos; la adaptación de las normas contables de valoración para tener en cuenta las excepcionales circunstancias de incertidumbre en los mercados; $y$, finalmente, la puesta en marcha de paquetes de estímulo presupuestario y fiscal encaminados a reanimar la demanda agregada. 
Esto indica que debe haber una causa endógena al propio sistema que las desorienta y las lleva a situaciones próximas al colapso. Las actuaciones discrecionales del Estado tienen el poder de desorientar o anular las leyes del mercado y que se lleven a cabo los ajustes necesarios. No sería, por tanto, infundado considerar las intervenciones discrecionales del Estado como ese elemento endógeno y desestabilizador del sistema financiero. En cualquier caso, un rediseño institucional que elimine las intervenciones discrecionales del Estado no podrá «eliminar» todo episodio de crisis, porque nunca se podrá eliminar el error empresarial y el error de apreciación que inducen a episodios de inestabilidad, incluso más allá de instituciones individuales. Sin embargo, el objetivo es mitigar su impacto y que lo que sea un proceso de crisis en una entidad sea una oportunidad de beneficio en otra de modo que se reduzca o se elimine el riesgo sistémico.

El debilitamiento de la estabilidad financiera por la intervención del Estado es una realidad que podría estar empezando a calar. El Banco de Inglaterra, por ejemplo, en el «Financial Stability Report» de octubre de 2008 (Banco de Inglaterra, 2008), discute cómo las ayudas públicas implícitas y explícitas a los bancos, crecientes a lo largo del siglo XX, han hecho que el sistema financiero se haya ido debilitando, y que la combinación de un proceso de desregulación pero manteniendo las garantías del Estado, se encuentren entre las causas de la rapidez y virulencia con que la actual crisis financiera se ha desarrollado. ${ }^{6}$

Por otra parte, el marco institucional creado para su salvaguarda se demostró ineficaz, así como las instituciones establecidas con el fin de vigilar y promover la estabilidad financiera. Ante un fondo de garantía de depósitos, un supervisor que vigila, y un banco central que provee liquidez, ¿quién puede tener interés en la calidad de la gestión, la solvencia y la liquidez de las entidades?, ¿y quién se puede extrañar, entonces, del colapso financiero y económico que ha traído la actual crisis económica mundial?

\footnotetext{
6 The banking crisis and the unprecedented interventions by national and international authorities will affect both the structure of the financial system and the incentives within it. The full consequences will take time to emerge. But recent events have highlighted the need for a fundamental rethink internationally of the appropriate safeguards against systemic risk. (Banco de Inglaterra, 2008, p. 41).
} 
Por último, habría que considerar que un fundamento conceptual para la revisión del paradigma dominante debía pretender la promoción, también en el sector financiero, de los principios del libre mercado, entendido como aquel sistema económico que reconoce la libre empresa, la prioridad de los acuerdos voluntarios, la propiedad privada y la total responsabilidad personal a la hora de asumir errores y aciertos. En un contexto de libre mercado, la intervención del Estado en el sistema financiero se debería limitar a asegurar la ley, los contratos, la propiedad privada y la estabilidad monetaria.

\section{Necesidad de revisar los presupuestos metodológicos}

La actual crisis económica mundial apunta al fracaso o a la insuficiencia del pensamiento económico dominante, pero también puede apuntar al fracaso o insuficiencia de los presupuestos metodológicos de la ciencia económica. Hay lagunas de conocimiento sobre los ciclos económicos y los fundamentos para alcanzar sistemas financieros sólidos, pero también plantea dudas sobre la efectividad de los aspectos metodológicos con los que cuenta en la actualidad la investigación científica en el campo de la economía para el estudio de la estabilidad financiera.

Apuntando algunos, puede que se esté otorgando excesivo valor a la capacidad analítica de los agregados perdiéndose las consideraciones individuales que suceden dentro de dichos agregados. La reflexión sobre la acción humana individual debería primar como el método más apropiado para estudiar y elaborar la teoría sobre los fenómenos económicos, optando por los agregados cuando las consideraciones individuales sean secundarias. Por otra parte, es un error metodológico asumir que toda información relativa a la acción humana pueda ser objetivamente presentable en agregados homogéneos, desestimando la naturaleza subjetiva de dicha información.

Íntimamente relacionado con el excesivo uso de agregados, se observa un abuso del lenguaje matemático con el objeto de dar una apariencia de homogeneización en la agregación de elementos que no son homogéneos. Si quitarle ningún mérito y utilidad 
a la formulación matemática, el lenguaje verbal recoge los fundamentos de los fenómenos económicos con más precisión y riqueza que el lenguaje matemático. Por otra parte, el formulismo matemático supone de hecho un impedimento para el avance del pensamiento económico al limitar el propio pensamiento solo a aquellas variables o relaciones que son medibles (Hayek, 1974). Se termina por estudiar solo aquello que es medible, o se trata de medir todo aquello sobre lo que se quiere estudiar, aunque dichas mediciones carezcan de significado.

Así mismo, la investigación actual en temas de estabilidad financiera está excesivamente centrada en el análisis empírico. La realidad puede confirmar hipótesis pero, por sí, no demuestra nada. La realidad hay que saber interpretarla con un modelo teórico previo. Lo importante es que la teoría que explique la realidad pase la prueba de la consistencia lógica, aspecto que no es prioritario en el desarrollo de estudios empíricos. Esta es una deficiencia metodológica importante en particular hoy donde nuevos avances tecnológicos y el efecto de la globalización hacen más necesario aún el descubrimiento de un modelo teórico que dé seguridad sobre lo que es un sistema financieramente estable.

Unido al abuso del método empírico está la utilización de modelos matemáticos como el principal cuando no el único modo de ver la realidad. La modelización reduce el espíritu crítico ante la tendencia humana a extrapolar la situación actual y proyectarla en el futuro. Lo que suceda mañana en el campo de la economía no puede conocerse "científicamente» hoy, pues depende en gran parte de un conocimiento e información que se está generado empresarialmente en todo momento. Cualquier predicción económica solo es de naturaleza cualitativa y relativa a la previsión de los desajustes y efectos de descoordinación en el mercado. 


\section{$\mathrm{V}$ \\ HACIA UNA ELIMINACIÓN DE LAS INTERVENCIONES DISCRECIONALES DEL ESTADO}

El paradigma dominante entiende que el Estado debe posibilitar la existencia del llamado coeficiente de caja para que el sistema bancario pueda expandir el crédito tanto como sea preciso y, así, atender más proyectos de inversión de los que permitiría el ahorro disponible. ${ }^{7}$ Para evitar el colapso de una banca con encaje fraccionario, se debe contar con un esquema de garantía de depósitos y un prestamista de última instancia públicos que mantengan la confianza en un sistema bancario incapaz de atender los depósitos a la vista. ${ }^{8}$ También se espera del Estado que rescate las instituciones que se consideren sistémicas. Además, el Estado a través de los bancos centrales debe intervenir en los tipos de interés y en la creación de dinero mediante una política monetaria discrecional.

No se está en contra de todos estos elementos. En este artículo se busca un diseño que contribuya a formar un sistema financieramente estable, y parar ello se reflexiona sobre un modelo en el que el Estado se abstrae de todo intento de actuación arbitraria y desestabilizadora sobre el sistema financiero, para limitarse a asegurar la existencia de las infraestructuras necesarias que garanticen el cumplimiento de la ley, los contratos y los principios generales del derecho. Cabe anticipar que la eliminación de la intervención discrecional del Estado supondría cambios radicales en el propio negocio del sector bancario, fomentando un

7 En general, el coeficiente de caja, también conocido como encaje bancario o coeficiente legal de reservas, es el porcentaje sobre los depósitos a la vista y sustitutos que un banco debe mantener en reservas líquidas (en la eurozona se rebajó del 2\% al $1 \%$ en enero de 2012). El multiplicador bancario es el inverso del coeficiente de caja y expresa la capacidad de la banca para expandir el crédito sin necesidad de ahorro voluntario previo. También expresa la capacidad de la banca de aumentar la oferta monetaria en función de la base monetaria. Con un coeficiente de caja del $2 \%$, la banca podría llegar a una expansión crediticia y de la oferta monetaria de 50 veces el importe de la base monetaria. En la práctica el efecto del multiplicador es más restringido.

8 Pese a este diseño del sistema financiero, la regulación prudencial a penas prestó a tención al riesgo de liquidez. 
tipo de negocio más estable por estar sujeto a unas leyes no distorsionadas de la economía y los principios generales del derecho, aunque menos rentable que el sistema bancario y financiero actual. Por lo que cabe también anticipar que un modelo de negocio bancario sin la actual intervención del Estado sería frontalmente opuesto por el poderoso e influyente sector bancario desde los campos de la política, los medios de comunicación y el mundo académico.

\section{La creación de dinero sin la actuación discrecionalidad del Estado}

El dinero es el bien económico con mayor capacidad para producir la coordinación o descoordinación social, ya que es el bien que normalmente se interpone en los intercambios en cualquier sociedad que haya superado el estado de trueque. Dada la importancia de la institución social del «dinero», una oferta monetaria estable y predecible es un elemento esencial para la estabilidad financiera.

El dinero no es algo que históricamente haya sido creado por los poderes públicos (lo mismo que los poderes públicos tampoco han creado el lenguaje o la cultura). ${ }^{9}$ Es una institución que se ha ido formando de forma espontánea de acuerdo con la experiencia milenaria de los intercambios. Por lo tanto, la eliminación de los poderes discrecionales del Estado sobre la creación del dinero no solo es posible, sino que sería el estado natural de las cosas. Lo poderes coactivos del Estado quedarían restringidos a proteger de su envilecimiento el bien económico que se acepte como dinero. Una tarea que no es menor, ya que desde el abandono gradual del "patrón oro» a lo largo del siglo XX, la experiencia ha demostrado que la creación discrecional de dinero fiduciario por los bancos centrales ha sido intrínsecamente inflacionaria, cuando no hiperinflacionaria, dependiendo de la calidad moral de los

\footnotetext{
9 Ver Menger (1871 [1997]), Capítulo VIII. Noonan (1957) permite ampliar el debate sobre la naturaleza del dinero.
} 
gobernantes ${ }^{10}$ Esto difícilmente podría ser de otra forma, ya que al tener la posibilidad de crear dinero fiduciario de la nada, sin otra restricción objetiva que la conciencia, y sin tener que organizar expediciones arriesgadas para conseguir oro o explotar costosos y peligrosos yacimientos auríferos, sería exigir conductas heroicas a los gobernantes que teniendo el inmenso poder de crear dinero, lo ignoraran y no lo ejercieran. De hecho, hasta ahora ningún gobierno lo ha ignorado y todos lo han ejercicio y, así, se constata como la inflación es una característica asociada a todas las economías con dinero fiduciario.

Si bien el dinero es un bien económico, no es ni un bien de consumo ni un bien de capital. Constituye en sí mismo una categoría distinta. ${ }^{11}$ De aquí se desprende que un incremento en la oferta monetaria no tiene ningún mayor valor social, por no ser ni un bien de capital ni un bien de consumo. La simple creación de dinero no supone una creación de riqueza. Todo lo contrario, supone una reasignación de riqueza entre los diferentes individuos de la sociedad. Es decir, con la creación de dinero de la nada (como sucede con la falsificación de billetes) se benefician aquellos que primero reciben el dinero recién creado, ya que tienen una mayor capacidad comparativa para pujar por los recursos escasos de la economía, y se perjudica al resto con un valor más diluido para sus tenencias de efectivo.

Desde planteamientos no intervencionistas, la creación de dinero fiduciario compatible con la salvaguardia de la estabilidad financiera debería estar sujeta a reglas estrictas y predecibles, o adoptar como dinero algún bien económico, el papel que a lo largo de la historia desempeñaron el oro y la plata.

${ }^{10} \mathrm{Ni}$ siquiera los descubrimientos de oro en América y su masiva entrada en Castilla supusieron el mismo efecto inflacionario que la política monetaria llevada a cabo por el banco central más responsable en el manejo de la base monetaria con dinero fiduciario.

11 Mises (1912) rebate la teoría de su maestro, Eugen von Böhm-Bawerk, que entendía que el dinero era un bien de capital. El argumento es evidente: en un proceso productivo, no cumplen la misma función el dinero que los bienes de capital auténticos. 


\section{Función restringida de los bancos centrales}

Aceptando que la creación de dinero esté sujeta a reglas estrictas y no discrecionales, la función de los bancos centrales sería asegurar la estabilidad monetaria (una estabilidad de la oferta monetaria similar a la que ofrecería el patrón oro) y proteger contra el envilecimiento aquel bien económico que haga la función de dinero. Junto a esta función de procurar y proteger la estabilidad de la oferta monetaria, los bancos centrales podrían seguir actuando como banco de bancos, banquero del Estado, y supervisión bancaria, pero se eliminarían sus funciones discrecionales sobre la creación de dinero: política monetaria y prestamista de última instancia.

Desde planteamientos no intervencionistas, los bancos centrales no podrían aumentar la base monetaria a su discreción, por lo que deberían abstenerse de tratar de controlar los tipos de interés de mercado. Dicho control, a través de las decisiones de política monetaria y operaciones de mercado abierto, se verían desde su naturaleza desestabilizadora al interferir en los intercambios voluntarios entre prestamistas y prestatarios a los que no les permite descubrir libremente la tasa de interés natural de equilibrio (Wicksell, 1966). La razón de ser de esta práctica intervencionista por parte de los bancos centrales se justifica con el fin de mantener una inflación de precios baja y estable, cuando precisamente la inflación de precios (además de las expectativas de inflación y de la demanda de dinero) es una consecuencia de los aumentos de la base monetaria.

Como sucede con todo precio intervenido, establecer precios por debajo de lo que sería su nivel natural de mercado provoca escasez al sobreestimular la demanda. En el caso del tipo de interés, una política monetaria acomodaticia tiende a generar una sobredemanda de crédito, que en el caso del diseño actual del sistema financiero y monetario puede ser atendida por la capacidad de crear crédito y dinero bancario que suplan la falta de oferta de ahorro real. Si para la articulación y progreso de una sociedad es necesario disponer de precios libres de mercado, esto debe ser más importante si cabe en el tipo de interés, el precio clave que articula la economía y los cálculos del coste de capital. 
Otra función con potencial desestabilizador de los bancos centrales es su función de prestamista de última instancia, ${ }^{12}$ que dejaría de ser necesaria en la medida en que se implantara un coeficiente de caja del 100\%, como se discute más adelante. De hecho, la función de prestamista de última instancia aparece para posibilitar el ejercicio de la banca con reserva fraccionaria y es posible en la medida en que los bancos centrales tienen la capacidad de crear ilimitadamente dinero fiduciario. Para algunos la función de prestamista de última instancia fue en sí el motivo de la creación de los bancos centrales (Rothbard, 1966).

Se justifica que la intermediación en los plazos es precisamente la función del sistema bancario. Esto es posible siempre que haya un fondo de garantía de depósitos que tranquilice a los depositantes y un prestamista de última instancia que, en casos de necesidad pueda acudir a solucionar situaciones financieramente inestables. Pero en una situación de libre de mercado, sin la intervención de las autoridades monetarias, la llamada «intermediación en los plazos» no sería posible llevarla a cabo sobre los balances bancarios.

\section{El negocio bancario sin privilegios del Estado}

Los bancos, en sus actividades de intermediación especializada en el crédito, de custodia de efectivo y gestión de tesorería, son imprescindibles para el desarrollo económico. Sin embargo, la actual crisis financiera ha sido testigo de cómo el diseño actual del sector bancario fuertemente intervenido y protegido por el Estado ha puesto a los pies de los caballos a la economía en su conjunto. Por otra parte, en un entorno económico general de economía de mercado, no debería resultar admisible que el sector

12 Existe la discusión sobre si la función de prestamista de última instancia puede ser ejercida por el mercado. Según esta opinión, el resto de participantes en los mercados monetarios e interbancarios estarían tan bien informados, sino mejor, de la condición de solvencia del resto de partícipes, por lo que están en una situación mejor para prestar solo a entidades ilíquidas, pero que demuestren ser solventes. Entidades potencialmente insolventes, de este modo, serían expulsadas del sistema bancario. 
bancario contase con semejante apoyo y protección del Estado para poder operar.

La eliminación de la protección estatal implicaría los siguientes cambios en el negocio bancario que supondrían la eliminación de sus características más inestables, ya qua ante mensajes plenamente creíbles de ausencia de protección del negocio bancario por el Estado, el mercado solo toleraría aquellos negocios estables y de riesgo bajo:

- Se eliminaría la banca con reserva fraccionaria, ya que los depositantes solo acudirían a aquellas entidades que demuestren plena capacidad para devolverles sus depósitos, incluso en situaciones de pánico.

- Como consecuencia, también se eliminaría la capacidad de la banca para crear sustitutos de dinero a través de la expansión crediticia, e igualmente, contraer la oferta monetaria cuando estos sustitutos de dinero se destruyan. Esto ajustaría el crédito bancario al ahorro real de la economía y reduciría significativamente los ciclos económicos (Mises, 1949; Huerta de Soto, 2009).

- Se eliminaría la propensión de la banca a invertir en operaciones de activo a largo plazo financiadas a corto plazo. Sin la protección estatal, los bancos deberían mostrar una estructura equilibrada de plazos para poder acceder a la financiación del mercado.

- Se eliminaría la propensión de la banca a mezclar los negocios de custodia de depósitos y gestión de tesorería con los negocios de riesgo crediticio y otros negocios de riesgo bancario. El mercado, sin la protección del Estado, rechazaría a aquellos custodios que expusiesen sus depósitos.

- Se eliminaría la doctrina de los así llamados bancos «too big to fail».

Habría que analizar si estas modificaciones en el negocio bancario son factores que refuerzan o debilitan la estabilidad financiera y la función de la banca en la economía. En cualquier caso, este modelo de negocio bancario sería frontalmente opuesto por los poderosos e influyentes lobbies bancarios, a quienes la 
situación de inestabilidad del sistema bancario actual no es algo ajeno o que les tomase por sorpresa. Como indicó Chuck Prince, ex-presidente y CEO de Citigroup, antes del estallido de la burbuja en el mercado de hipotecas sub-prime (Financial Times, 10 de julio de 2007) ${ }^{13}$ : "When the music stops, in terms of liquidity, things will be complicated. But as long as the music is playing, you've got to get up and dance. We're still dancing». Entienden bien que el negocio bancario es intrínsecamente inestable, pero es tan conveniente a sus intereses que no lo abandonarán sin lucha.

Por último, toda institución de crédito, para que sea considerada como tal, ha de poner la seguridad de sus depósitos como su primera prioridad deontológica. Esta certeza se debilita en tiempos de crisis, y una pregunta que puede aflorar en la sociedad, en esos mismos tiempos de crisis, es para qué y a quién sirven los bancos (accionistas, depositantes, acreedores, prestatarios). Cuando la respuesta no es una clara orientación a la seguridad de sus depósitos, la misma concepción del negocio bancario falla.

\section{VI \\ LA INSTITUCIÓN DE LA BANCA CON RESERVA FRACCIONARIA}

Un estudio más detenido merece la práctica de la banca con reserva fraccionaria por su impacto en la estabilidad financiera. Como vimos, una banca con reserva fraccionaria es posible por la cobertura legal que ofrece el coeficiente de caja, se ha visto enormemente favorecida por la existencia de dinero fiduciario que se puede crear de la nada a voluntad de las autoridades monetarias, y ha encontrado su posibilidad operativa gracias al apoyo implícito de las autoridades monetarias de esta práctica mediante su función de prestamista de última instancia. Con el tiempo, el encaje legal se ha ido reduciendo y en la actualidad la reducción trata de alcanzar un encaje legal mínimo que sea, en la práctica, el

13 Chuck Prince presentó su dimisión el 4 de noviembre de 2007, justo antes de que se iniciase el colapso de Citigroup. La prensa de entonces indicó que se retiró con una paga en torno a los 38 millones de dólares estadounidenses. 
encaje técnico que los bancos mantendrían de todas formas para hacer frente a su operativa en efectivo. En estas circunstancias, la expansión del crédito bancario no encuentra más límites que el propio coste de la financiación y los requerimientos regulatorios de capital de los bancos.

Una banca que operase sin reserva fraccionaria, por la exigencia del cumplimiento de los contratos de depósito, y que por tanto eliminase las posibilidades de la banca para sobre-apalancar la economía sería, sin duda, un elemento que colaboraría a salvaguardar la estabilidad financiera. Es cada vez más una doctrina aceptada internacionalmente a medida que avanza el estudio de la actual crisis financiera, que la causa de los ciclos económicos se encuentra, precisamente, en la expansión desmesurada del crédito bancario. Falta concluir en estos estudios que esto es posible e incluso se exacerba por la práctica de la banca con reserva fraccionaria. Por otra parte, es una obviedad que la banca con reserva fraccionaria es financieramente inestable, dado el riesgo real de una retirada masiva de los depósitos a la vista, cómo sucedió de forma global y sistémica durante los meses de septiembre y octubre de 2008. Confianza en los bancos que solo se pudo recobrar con la garantía por parte de los gobiernos de la práctica totalidad de los depósitos bancarios.

La práctica de la reserva fraccionaria también se puede ver como una vulneración legal de los derechos de propiedad promovida por el Estado de «la institución del depósito» del derecho romano (Huerta de Soto, 2009). En principio, los depósitos irregulares de dinero que reciben las entidades de crédito y que son exigibles a la vista, deberían mantenerse íntegramente a disposición de los depositantes, salvo que se pactara lo contrario.

El ejercicio de la banca sin reserva fraccionaria no es una contribución novedosa. Es, por el contrario, la forma en la que originariamente se entendió un ejercicio honesto del negocio bancario. Fueron las presiones a través de los siglos de banqueros y gobernantes, ante las posibilidades de mayor lucro y disponibilidad de recursos, las que han ido creando las bases operativas, legales e intelectuales que lo justifican y posibilitan, y que, por último, forzaron el abandono del patrón oro. Históricamente siempre ha habido una tendencia fuerte a vulnerar el derecho de propiedad 
de los depositantes sobre sus depósitos bancarios. Por una parte, los gobiernos responsables de la aplicación de la ley han visto en esta práctica una posibilidad de financiar sus gastos públicos sin la necesidad de tener que tomar medidas impopulares y recaudar nuevos impuestos. Por otro, los banqueros han visto en esta práctica un modo de obtener mayores ingresos. Desde que hay memoria de la existencia de bancos, los banqueros han encontrado tentador prestar el dinero que han recibido en depósito.

Se argumenta a favor de la práctica de la banca con reserva fraccionaria que permite a los bancos retribuir depósitos a la vista, que de lo contrario devengarían comisiones de custodia y gestión de tesorería. Habría que discernir qué resulta más oneroso si pagar comisiones por la gestión y custodia de los depósitos a la vista, o soportar los costes de la inflación y las recesiones económicas. Este es un tema de educación financiera de la población en general. ${ }^{14}$ También se argumenta que sin la reserva fraccionaria los bancos serían menos rentables. Esto efectivamente es así, pero quizá hay margen para reducir la rentabilidad con el objetivo de conseguir un sistema bancario sólido, estable y coherente. De hecho, la rentabilidad de la banca y las retribuciones a sus directivos son tan altas que durante estos últimos años las mentes más brillantes de las universidades siempre que han podido han preferido ser contratadas por instituciones de crédito y desempeñar allí sus funciones, que emplearse en otras actividades que fuesen socialmente más provechosas.

Por último, se justifica la práctica de la banca con reserva fraccionaria por la mayor eficiencia económica que supone la expansión del crédito bancario por encima del ahorro disponible. Gracias a esta expansión crediticia la humanidad ha podido avanzar en el último siglo como no lo había hecho antes. Se atribuye el éxito del progreso al crédito bancario y no a las mentes

${ }^{14}$ Una reforma que contribuya a salvaguardar la estabilidad financiera ha de considerar también una sólida formación financiera de los ciudadanos. Es parte del deber de los organismos públicos y privados fomentar la formación de la sociedad en temas de finanzas y economía sobre la base de la realidad humana y el conocimiento de sus deberes y responsabilidades. Es también el deber de la ciudadanía entender los procesos de libre mercado para el crecimiento y la eficiencia económica. 
que hicieron posible este progreso. Habría que pensar si este mayor progreso económico no es fruto, más bien, del aumento de la población con formación y no tanto del crédito bancario inducido. Por otra parte, esta argumentación cae de nuevo en el error de confundir dinero con riqueza y no tiene en cuenta el coste de los ciclos económicos generados por una expansión desmesurada del crédito bancario.

\section{VII \\ LA BANCA LIBRE}

La «banca libre», el sistema bancario sin banco central en el que la emisión y depósitos de dinero están a cargo de bancos privados, es una línea de investigación que adquirió en los trabajos de Hayek (1978) una particular atención, aunque sobre propuestas ampliamente discutidas. La banca libre es un sistema monetario que hoy se desconoce en la práctica, a pesar de que siglos atrás era ampliamente seguido en casi todos los países cuando estaba vigente el patrón oro. Los beneficios que se obtenían del señoreaje de emisión indujeron a los Estados a crear sus propios bancos a los que de forma paulatina se les otorgó el monopolio de la emisión, o se otorgaron estos privilegios especiales de emisión a algún banco comercial privado.

El debate surge sobre la posibilidad práctica y teórica de una banca libre con reserva fraccionaria. Mises, desde el inicio (Mises, 1912), ya introdujo la idea de que una banca libre exigía un coeficiente de caja del 100\%. Sin embargo, algunos economistas han propuesto una banca libre con reserva fraccionaria (Lawrence White, Goerge Selgin, o Stephen Horwitz). El ejercicio de la banca con reserva fraccionaria, se considere libre o no, conduce a la necesidad de un banco central y a la intervención del Estado en el sistema financiero, es inflacionista y generadora de ciclos económicos (Huerta de Soto, 2009). ${ }^{15}$ Por otra parte, una banca con

15 Ver , Capítulo VIII: «Podemos, por tanto, concluir que ... la discusión teórica y práctica debe plantearse no en los términos tradicionales [entre "escuela bancaria", defensora del banco central, y "escuela monetaria", defensora de la banca libre], sino entre 
encaje fraccionario, sin el respaldo de un banco central, está llamada a desaparecer, ya que el propio mercado eliminará aquellos bancos que no tengan una cobertura plena de sus depósitos.

\section{VIII \\ LA REGULACIÓN DE LAS INSTITUCIONES PRIVADAS DE CRÉDITO}

En cuanto a la regulación de las instituciones privadas de crédito en un entorno sin actuaciones discrecionales del Estado, una sola norma sería básica y necesaria, y que ni siquiera tiene que ser parte de una regulación bancaria: la prohibición de la práctica de banca con reserva fraccionaria sobre aquello que el mercado haya decidido utilizar como dinero, salvo que haya acuerdo expreso en contrario. Esta norma induciría de manera natural a los bancos a tener casados los vencimientos de activos y pasivos. La ausencia de prestamista de última instancia y de garantías públicas de los depósitos, así como la posibilidad de quiebras bancarias sin rescate estatal, crearían los incentivos correctos que fomentasen la solvencia bancaria a través de la disciplina de mercado. Con una norma que prohibiese la práctica de la banca con reserva fraccionaria en un entorno de ausencias de garantías públicas, el resto de posibles problemas que amenazan la estabilidad financiera también quedarían resueltos, es decir, el riesgo sistémico por el colapso de los sistemas de pagos, el riesgo moral de los ahorradores, el riesgo de información asimétrica en el riesgo de contraparte, y los esquemas de incentivos a directivos.

El supervisor prudencial debería contar con autoridad y una sólida reputación profesional, sin poderes discrecionales, y centrado en la seguridad jurídica del negocio bancario y el cumplimiento de los contratos. En particular, su función sería velar

dos sistemas radicalmente distintos, a saber: o bien un sistema de banca libre sometida a los principios tradicionales del derecho (coeficiente de caja del 100 por cien) ...; o bien un sistema que permita el ejercicio de la banca con reserva fraccionaria y del que, de manera inevitable, habrá de surgir un banco central prestamista de última instancia y controlador de todo el sistema financiero». 
para que los bancos dispongan de lo que deben mantener disponible a la vista. Esto no quiere decir que vaya a ser una tarea fácil de lograr, sino una actividad extremadamente compleja en un entorno de creciente innovación financiera y tecnológica. El ingenio humano alcanzará límites impensables para tratar de saltarse esta norma y lucrarse como ha sido la constante histórica con los fondos depositados para su custodia. La ingeniería financiera ha demostrado en las últimas décadas hasta qué punto la creatividad de la banca será todo un reto para la labor del supervisor. Así mismo, el supervisor debería velar por que la información que los bancos suministren al mercado sea veraz, limitándose a establecer los controles necesarios para que se dé la correcta valoración de activos y el reconocimiento de beneficios y pérdidas, de acuerdo con unos principios de contabilidad generalmente aceptados.

Como vimos, la doctrina dominante entiende que la regulación financiera se debe fundar en la identificación explícita de «fallos de mercado» que dicha regulación buscará corregir. Sobre el diseño de un sistema financiero intervenido, intrínsecamente inestable, se espera que el regulador y supervisor financiero realicen lo imposible: mantenerlo estable. Desde una postura no intervencionista, los fallos del mercado son oportunidades de perfeccionamiento por el propio mercado siempre que se le deje actuar en libertad, en plena asunción de las responsabilidades individuales y en un marco de seguridad jurídica. La regulación discrecional de los bancos no es una actividad carente de riesgo para la economía, y es discutible que sea la solución más eficaz para prevenir situaciones de inestabilidad financiera. Por una parte, la propia regulación es en muchas ocasiones la causa de los problemas que llevan a la pérdida de la estabilidad financiera; por otra, las agencias de regulación raramente identifican los problemas antes de que estos sucedan, por lo que no serán la solución más apta para adelantarse a los así llamados fallos de mercado. 
IX

\section{CONCLUSIÓN: EFECTOS SOBRE LA ESTABILIDAD FINANCIERA DE LA ELIMINACIÓN DE LA INTERVENCIÓN DISCRECIONAL DEL ESTADO}

Es necesario un pensamiento alternativo ante las situaciones que estamos viviendo y, en este sentido, este artículo apunta hacia nuevos enfoques. El actual debate internacional sobre estabilidad financiara está dominado por escuelas de pensamiento que ven necesaria la intervención del Estado para fomentar, salvaguardar y recobrar la estabilidad financiera. Este artículo, por el contrario, parte de las intervenciones discrecionales del Estado en los sistemas monetarios y financieros como el principal riesgo al que se enfrenta una economía para fomentar, salvaguardar y recobrar la estabilidad financiera. Las funciones públicas de política monetaria, prestamista de última instancia y garante, implícito o explícito, del sector financiero son revisadas como una de las principales causas endógenas del riesgo sistémico.

Propuestas alternativas que entren frontalmente en conflicto con la doctrina pacíficamente aceptada por la totalidad de la teoría económica dominante, así como por buena parte de la población educada e informada de acuerdo con esta doctrina, reciben muy poca atención. Un ejemplo claro es la propuesta de un coeficiente de caja del $100 \%$. Este tipo de propuesta muy rara vez es discutida o siquiera considerada por la teoría económica dominante, aún cuando los beneficios directos para la estabilidad financiera son evidentes y ha habido célebres economistas y premios Nobel de Economía que la han propuesto. Este artículo ha pretendido ser un contrapunto a este silencio.

Como se ha discutido en el artículo, no es infundado considerar las intervenciones discrecionales del Estado como fuente de inestabilidad financiera. Los beneficios teóricos de un primer análisis son importantes. Ante todo, se promueve la entrada del libre mercado en el sector financiero, fuertemente intervenido en la actualidad. En un entorno de libre mercado, los bancos centrales quedan reducidos a lo que legitima la existencia del Estado: asegurar la estabilidad monetaria, hacer cumplir la Ley, y respetar los derechos de propiedad y los contratos. Mantener la 
discreción del Estado para manipular la oferta monetaria, o la intervención necesaria del Estado para evitar que colapse un sistema bancario con reserva fraccionaria, solo consigue que la sociedad siga siendo dependiente de la intervención de los poderes públicos en el sistema financiero. Sin embargo, los obstáculos principales para su implantación no son técnicos, sino políticos, sociales y culturales.

La eliminación del encaje fraccionario encontraría una de sus mayores resistencias en los propios banqueros que verían seriamente disminuida la rentabilidad, así como eliminada la capacidad de sus bancos de crear dinero y, por tanto, de ejercer poder. La eliminación de los privilegios estatales que dan cobertura legal al encaje fraccionario pondría fin a una larga lucha y conquista de décadas por parte de los banqueros de alcanzar mayores cotas de poder económico y político. La presión del sector bancario para evitar esta medida sería muy fuerte. Sus armas volverían a ser la presión política, mediática e intelectual desde el mundo académico. La medida de la eliminación del encaje fraccionario también encontraría resistencia en el propio público ahorrador y supondría un cambio cultural importante que implicaría que los bancos, en su gestión de pagos y custodia de efectivo, no solo no podrían pagar intereses, sino que tendrían que cobrar comisiones para que la actividad fuese viable.

La eliminación de la reserva fraccionaria y el establecimiento de un coeficiente de caja del $100 \%$, ante todo, sería el elemento clave para la eliminación del principal riesgo para la estabilidad financiera: la expansión del crédito bancario por encima del ahorro voluntario. Por otra parte, forzaría a la banca a casar plazos. En particular, fuerza a la banca a dejar la práctica de endeudarse a corto plazo para prestar a largo plazo, reforzando la estabilidad financiera. Forzaría a la banca a separar la actividad crediticia de riesgo de impago y quiebra, de la actividad de gestión y custodia de efectivo. Permitiría la eliminación del banco central como prestamista de última instancia, e introducir medidas para evitar la existencia de entidades too-big-to-fail, reforzando la disciplina de mercado. Por estar cubiertos los depósitos y sustitutos de dinero al 100\%, el riesgo cubierto por los esquemas de garantías de depósitos quedaría reducido al riesgo de fraude. Por 
último, simplificaría enormemente la regulación bancaria. Solo una norma sería básica: prohibir la existencia de banca con reserva fraccionaria. Una norma, sin embargo, muy compleja de supervisar en la práctica y que requeriría una alta competencia profesional del supervisor.

La medida necesaria más simple, desde un punto de vista técnico, es establecer normas fijas de política monetaria. Tan solo se requeriría una disposición que así lo determine y un diseño institucional adecuado que asegurase su cumplimiento. Es solo, por tanto, un tema de voluntad politica. Sin embargo, es aquí donde radica su gran dificultad de implantación que, en el momento de escribir, la hace prácticamente impensable. La doctrina económica y monetaria dominante da la razón a los poderes públicos, lo que ha hecho que se pueda decir que propuestas de política monetaria que tengan como principal foco de atención la estabilidad y predictibilidad de la oferta monetaria estén incluso fuera del espectro político.

Por su parte, el establecimiento de reglas no discrecionales por las que se regiría la política monetaria y por tanto del crecimiento de la base monetaria, ante todo, proporcionaría estabilidad y racionalidad económica al sistema de precios, lo que beneficia la estabilidad financiera. Fomentaría que el mercado pueda contar con un bien económico que cumpla las funciones del dinero. Un dinero no inflacionario que sea aceptado por el mercado. Por último, fomentaría la justicia entre los distintos miembros de la sociedad, en el sentido de que nadie ve diluidos sus ahorros en favor de otros vía la inflación discrecional de la base monetaria. Junto a aspectos monetarios y económicos, la estabilidad financiera también se construye sobre la base de una sociedad moralmente sólida.

Por último, una nota final de cautela. Un estudio sobre el proceso de eliminación de la fuerte intervención del Estado sobre el sistema financiero, que no es el objeto de este artículo, desaconsejaría su eliminación de forma abrupta. Como todo cambio radical y profundo, se debería hacer de manera organizada, en un proceso de transición muy delicado, con el riesgo adicional que situaciones de apoyo gubernamental pervivan en un proceso general de liberalización, creando las bases para situaciones de inestabilidad mucho más perniciosas de las que se trataban de evitar. 


\section{REFERENCIAS BIBLIOGRÁFICAS}

BAgeHot, W. (1873): Lombard Street: A Description of the Money Market, Londres: H.S. King, www.econlib.org/library/Bagehot/ bagLom.html.

BANCO DE INGLATERRA (2008): Financial Stability Report, October 2008, Londres: Bank of England, http:/ / www.bankofengland.co. uk/publications/fsr/2008/fsrfull0810.pdf.

FrIEDMAN, M. (2006): The optimum quantity of money, New Brunswick, N.J.: Aldine Transaction.

Goodhart, CH., y Shoenmaker, D. (1995): «Should the Functions of Monetary Policy and Bank Supervision be Separated?», Oxford Economic Papers, vol. 39.

Guillory, G. (2005): «What Are You Calling Failure?» en: Daily Article, The Mises Institute, 5 de mayo, www.mises.org/ story/1806.

Hayek, F.A. von (1974): «The Pretence of Knowledge» Discurso de entrega del premio, The Sveriges Riksbank Prize in Economic Sciences in Memory of Alfred Nobel 1974, Estocolmo, http:/ / nobelprize.org/nobel_prizes/economics/laureates/ 1974/hayek-lecture.html.

- (1978): Denationalization of Money. The Argument Refined: an Analysis of the Theory and Practice of Concurrent Currencies, Londres: Institute of Economic Affairs.

Huerta de Soto, J. (2009): Dinero, crédito bancario y ciclos económi$\cos , 4$ a edición, Madrid: Unión Editorial.

Menger, C. (1871 [1997]): Grundsätze der Volswithchaftslehre, Wilhelm Braumüller, Viena. Traducción española: Principios de Economía Política, Madrid: Unión Editorial.

Mises, L. VON (1912 [1961]): The Theory of Money and Credit, traducción al español, Teoría del dinero y crédito, Barcelona: Zeus. Disponible en inglés: http://mises.org/books/tmc. pdf?bcsi_scan_44441617FDB44D6E=ydXWEBF4tC 67jfpcTaayCgoAAACu+vUF\&bcsi_scan_filename $=$ tmc. pdf.

- (1949 [2004]): Human action, a treatise in economics, traducción al español: La acción humana, tratado de economía, 8. edición, Madrid: Unión Editorial. 
NoOnAn, J.T. (1957): The Scholastic Analysis of Usury, Harvard, MA: Harvard University Press.

RothbARD, M.N. (1966): Man, economy and State: a treatise on economic principles, The Ludwig von Mises Institute, Auburn, Alabama, www.mises.org.

SCHINASI, G.J. (2006): Safeguarding financial stability: theory and practice, Washington: International Monetary Fund.

WiCKSELL, K. (1966): «The natural rate of interest», en: Monetary theory and policy, ed. Richard A. Ward, Scranton, Penn.: International Textbook, pp. 233-248. 